\title{
Degradation of Polyethylene Plastic by Non-Embedded Visible-Light Iron-Doped Zinc Oxide Nanophotocatalyst
}

\author{
Received 12 January, 2021; revised 16 February, 2021; accepted 19 February, 2021
}

\author{
Maryam Ziaa ${ }^{a, \dagger}$, Sulaiman Faisal ${ }^{a, b, \dagger}$, Dilawar Farhan Shams ${ }^{c}$, Farida Anjum ${ }^{d}$, Mehk Saeed ${ }^{a}$, ZiaUllah Shah ${ }^{\circledR}$ \\ and Akhtar Nadhman ${ }^{a, *}$ \\ a Deparment of Biotechnology, Institute of Integrative Biosciences, CECOS University, Hayatabad, Phase VI, Peshawar \\ 25000, Pakistan \\ bInstitute of Biotechnology and Genetic Engineering, University of Agriculture, Peshawar 25000, Pakistan \\ cDepartment of Environmental Sciences, Abdul Wali Khan University, Mardan 23200, Pakistan \\ dDirectorate of Soil and Plant Nutrition, Agricultural Research Institute Tarnab, Peshawar 25000, Pakistan \\ e Department of Pharmacy, Institute of Integrative Biosciences, CECOS University, Hayatabad, Phase VI, Peshawar \\ 25000, Pakistan \\ †Contributed equally to this work as first author.
}

*Corresponding author E-mail: nadhman@cecos.edu.pk

\begin{abstract}
This study investigated the photocatalytic degradation of pure low-density polyethylene (LDPE) and commercial-grade polyethylene (PE) films with iron-doped zinc oxide $(\mathrm{Fe}-\mathrm{ZnO})$ nanoparticles under visible light. The study was particularly focused on the role of reactive oxygen species (ROS) and the types of plastic degradation. The Fe- $\mathrm{ZnO}$ were synthesized using the co-precipitation method and characterized by TEM and XRD. Degradation of $6.35 \mathrm{~cm}^{2}$ films of LDPE and commercial grade PE was tested under artificial LED light $(84 \mathrm{~lm} / \mathrm{W})$ and dark in Fe-ZnO suspensions of $10 \mathrm{ml}$ having concentrations ranging from 10 to $1000 \mu \mathrm{g} / \mathrm{ml}$. The results showed a maximum weight reduction of $13.8 \%$ for pure LDPE films at $200 \mu \mathrm{g} / \mathrm{ml}$ and $15 \%$ for commercial grade PE at $1000 \mu \mathrm{g} / \mathrm{ml}$ in 14 days. In comparison, no weight reduction was observed in the dark, which confirmed that the degradation was induced by the production of ROS moieties in visible light i.e., singlet oxygen (30.11 \%), hydroxyl ions $(30.45 \%)$, and hydroxyl radicals $(39.34 \%)$. The degradation was further confirmed by FTIR with the formation of alcohols and alkenes and SEM analysis that showed visible cracks in both LDPE and PE. The study unveiled Fe-ZnO nanoparticles as an efficient substitute to degrade polyethylene under visible light.
\end{abstract}

Keywords: Low-density polyethylene, ZnO, Nanoparticles, Plastic degradation, Reactive oxygen species

\section{Introduction}

Plastics are synthetic polymers that are tough to degrade in ambient environmental conditions due to their high molecular mass density resulting in a buildup in the environment [1-3]. Worldwide, plastic waste production is estimated to exceed 275 million metric tons of which 4.8 to 12.7 million metric tons end up in the oceans every year while 22 to $43 \%$ is disposed of in landfills and only $9 \%$ is recycled [4]. One of the most commonly used plastic is low-density polyethylene (LDPE) [5] which accounts for $60 \%$ of the total plastic production as plastic bags and therefore constitutes a major fraction in municipal solid waste piling up in land and marine ecosystems and increasingly recognized as a major ecological challenge [6-8]. LDPE is a linear or branched monomer of ethylene molecules that are extensively used in packaging due to excellent mechanical properties, hydrophobicity, light weightiness, and low-cost $[9,10]$. Due to their resistance to biodegradability under natural environmental conditions, they tend to remain in the environment for hundreds of years $[11,12]$ and therefore require efficient methods for degradation and removal.
Most of the research carried out on the degradation of LDPE has centered on biodegradation with microbes, however, the process is slow and therefore attention has been diverted to other modes of degradation. These include thermal, catalytic, mechano-chemical, and photooxidative degradation. Among these methods, photocatalytic degradation is considered the most capable $[13,14]$. For photocatalysis of plastics, organic or traditional photosensitizers and nanoparticles have been used. Traditional photosensitizers have limitations such as lack of long-wavelength absorption band, hydrophobicity, payload leaking, use of high concentrations, and low reactive oxygen species (ROS) production [15-17]. Comparatively, nanoparticles have been found more advantageous because of higher activity at low concentrations and dormancy in dark conditions [18]. These characteristics make nanoparticles highly applicable for the degradation of plastics [19] especially for polyethylene (PE) [20].

Previously, nanoparticles have been used for plastic treatment, however, most of the work has been on embedding or grafting the nanoparticles in polyethylene films [21-24]. Others have used nanoparticles such as silicon dioxide $\left(\mathrm{SiO}_{2}\right)$ in combination with microorganisms 
[25]. Photocatalytic degradation has also been researched with titanium oxide $\left(\mathrm{TiO}_{2}\right)$ but in UV light and also combining dyes to enhance the photocatalytic activity [26]. To our knowledge, no study has been carried out using non-embedded visible light excited zinc oxide $(\mathrm{ZnO})$ nanoparticles for polyethylene degradation.

$\mathrm{ZnO}$ is one of the promising choices because they are a low-cost and environmentally friendly semiconductor $[27,28]$ with a wide bandgap energy of $3.3 \mathrm{eV}$ at room temperature [29] with high excitation energy of $60 \mathrm{eV}$ [30]. However, a key limitation of $\mathrm{ZnO}$ is its activation under UV light. A potential strategy to overcome this problem is doping with transition metals especially iron [31,32]. Iron is considered the best dopant because iron $(\mathrm{Fe})$ exists in two possible oxidation states and it is a chemically stable element. The two oxidation states of iron are; $\mathrm{Fe}^{2+}$ and $\mathrm{Fe}^{3+}$ with ionic radii of 0.78 and $0.64 \AA$, respectively, which is close to the ionic radius of $\mathrm{Zn}^{2+}(0.74 \AA)$. This makes it easier for Fe to enter into $\mathrm{Zn}$ lattice sites without disturbing the crystal structure of $\mathrm{ZnO}$ and it contributes to improving its photocatalytic properties and conductivity [33]. Photocatalytic degradation with doped $\mathrm{ZnO}$ especially iron doping could be more efficient than UV activated nanoparticles such as $\mathrm{TiO}_{2}, \mathrm{SiO}_{2}$ because iron doped $\mathrm{ZnO}$ could be activated in visible light due to its ability to absorb a larger portion of the visible portion of the electromagnetic spectrum [34]. Visible light helps in more degradation via generating more free radicals. The ground state of Fe produces $\mathrm{ROS}$ and holes in the $\mathrm{ZnO}$ matrix. The ground state of Fe has low oxidative ability than the valance state to Fe. These electron-hole pairs react with adsorbed oxygen $\left(\mathrm{O}_{2}\right)$ molecules and cause the production of different $\mathrm{ROS}$ i.e. $\mathrm{OH}$ and $\mathrm{O}_{2}$. The objective of this study was to investigate iron-doped $\mathrm{ZnO}$ nanoparticles (Fe- $\mathrm{ZnO}$ ) for the degradation of PE with a focus on the role of ROS generated from the surface of these nanoparticles.

\section{Experimental details}

\subsection{Chemicals and reagents}

Zinc sulfate $\left(\mathrm{ZnSO}_{4}\right)$, iron sulfate $\left(\mathrm{FeSO}_{4}\right)$, potassium hydroxide $(\mathrm{KOH})$, xylene, and ethanol were purchased from Scharlau, Spain; di-phenyl iso-benzofuran (DPBF) dye, sodium azide, mannitol, and dimethyl sulphoxide from Sigma-Aldrich, USA; and pure LDPE granules and commercial grade PE bags from the local market.

\subsection{Synthesis of nanoparticles}

$\mathrm{Fe}-\mathrm{ZnO}$ were synthesized by the co-precipitation method. $\mathrm{ZnSO}_{4}$ and $\mathrm{FeSO}_{4}$ were used as precursor salts and $\mathrm{KOH}$ as a reducing agent [35]. $\mathrm{KOH}$ was added drop-wise to $100 \mathrm{mM}$ aqueous solution of $\mathrm{ZnSO}_{4}$ and $11 \mathrm{mM}$ solution of $\mathrm{FeSO}_{4}$ in the molar ratio of 2:1 [36]. The reaction was undertaken at $65^{\circ} \mathrm{C}$ for $2 \mathrm{~h}$. The synthesized nanoparticles were washed with water and ethanol at $10,000 \mathrm{rpm}$ for 15 min each and were dried overnight at $90^{\circ} \mathrm{C}$. Annealing was then done for $18 \mathrm{~h}$ at $200{ }^{\circ} \mathrm{C}$.

\subsection{Characterization of nanoparticles}

For the characterization of synthesized nanoparticles, particle size and morphology were confirmed by TESCAN electron microscopy (TEM, Joel MIRA3, Japan). The crystalline structure was analyzed by X-ray diffraction (XRD, JDX-3532 JEOL, Japan).

\subsection{Synthesis of pure LDPE films}

Pure LDPE films were synthesized by immersing $1 \mathrm{gm}$ of pure LDPE granules in $20 \mathrm{ml}$ of xylene $\left(\mathrm{C}_{8} \mathrm{H}_{10}\right)$ and heated on a hot plate at $150{ }^{\circ} \mathrm{C}$ overnight. The dissolution of LDPE granules in xylene formed solid LDPE material, which was then washed with water and ethanol to remove excess xylene and dried for 3 days. LDPE sheet was cut into films $\left(6.35 \mathrm{~cm}^{2}\right)$ and used for further experiments.

\subsection{Photo-thermal activity}

The photo-thermal activity was performed to check the variation in temperature of nanoparticles suspension after exposure to light. Nanoparticle suspension was taken in a tube and temperature was checked using a digital thermometer. The tube was exposed to LED light $(84 \mathrm{~lm} / \mathrm{W})$ for $5 \mathrm{~min}$ and the temperature was checked after every $1 \mathrm{~min}$. The procedure was repeated 2-3 times to confirm the change in the temperature.

\subsection{Quantification and identification of ROS}

For quantification of ROS, $1 \mathrm{ml}$ of DPBF dye solution (prepared by dissolving $0.0054 \mathrm{~g}$ of DPBF in $50 \mathrm{ml}$ of ethanol) and $1 \mathrm{ml}$ of nanoparticles suspension were added in a sealed quartz cuvette. The cuvette was exposed to sunlight with an infrared filter for $30 \mathrm{~s}$ and then the absorbance was checked at $410 \mathrm{~nm}$ using a spectrophotometer after every $30 \mathrm{~s}$ for up to $5 \mathrm{~min}$ [37].

For identification of different ROS produced, nanoparticles were treated in three groups of chemicals used for scavenging ROS i.e. 0.001 M sodium azide $\left(\mathrm{NaN}_{3}\right)$ for singlet oxygen, $5 \%$ DMSO for hydroxyl ions, and $0.001 \mathrm{M}$ mannitol for hydroxyl [38]. Each of these treatments containing the scavenger chemical, nanoparticle suspension, and DPBF were taken in individual cuvettes. While $\mathrm{Fe}-\mathrm{ZnO}$ suspension, DPBF, and a combination of the three scavengers were used as control. All the cuvettes were exposed to LED light for $5 \mathrm{~min}$. Absorbance was checked at $410 \mathrm{~nm}$ before and after exposure to light. The absorbance values were used for the percent calculation of possible ROS type produced.

\subsection{Experimental setup}

The synthesized LDPE films $\left(6.35 \mathrm{~cm}^{2}\right.$ size $)$ were weighed and suspended in $10 \mu \mathrm{g} / \mathrm{ml}, 100 \mu \mathrm{g} / \mathrm{ml}$, and $200 \mu \mathrm{g} / \mathrm{ml}$ nanoparticle suspension $(10 \mathrm{ml})$ and also in only deionized water as a control. The experiments were carried out under LED light in a box with six LED bulbs (combined flux of $84 \mathrm{~lm} / \mathrm{W}$ ) and also in dark for 14 days and constantly monitored. After 14 days, LDPE was taken out from the nanoparticle suspension and dried in the oven at $90{ }^{\circ} \mathrm{C}$ for $4-5 \mathrm{~h}$. After drying, different analysis was performed to confirm LDPE degradation. In a follow-up experiment, a commercial-grade PE bag was taken from the local market and cut into squared films of the same size as LDPE (6.35 $\mathrm{cm}^{2}$ ) and added to nanoparticle suspensions $(10 \mathrm{ml})$ with concentrations of 100,500 , and $1000 \mu \mathrm{g} / \mathrm{ml}$ while PE films in deionized water were taken as control. The same experimental conditions were used as for the synthesized LDPE.

\section{Photo-degradation analysis}

To analyze the weight reduction in plastic, an analytical balance was used with a sensitivity of $0.1 \mathrm{mg}$ [26]. Weight reduction (\%) was calculated using Eq. (1).

$$
\text { Weight loss }(\%)=\frac{\text { Initial weight }- \text { Final weight }}{\text { Initial weight }} \times 100
$$

To observe the cracks, roughness, and change in surface morphology of both LDPE and commercial grade PE films, a scanning electron microscope (SEM) was performed before and after photo-degradation using JEOL JSM-5920, Japan. Fourier-transform infrared spectroscopy (FTIR) analysis was performed to confirm the formation of different functional groups after degradation of LDPE [39]. Samples were taken before and after exposure to light to indicate the variation in FTIR peaks after degradation.

\section{Data analysis}

All the experiments were performed at least three times to ensure their reproducibility. The data from all these experiments were pre- 


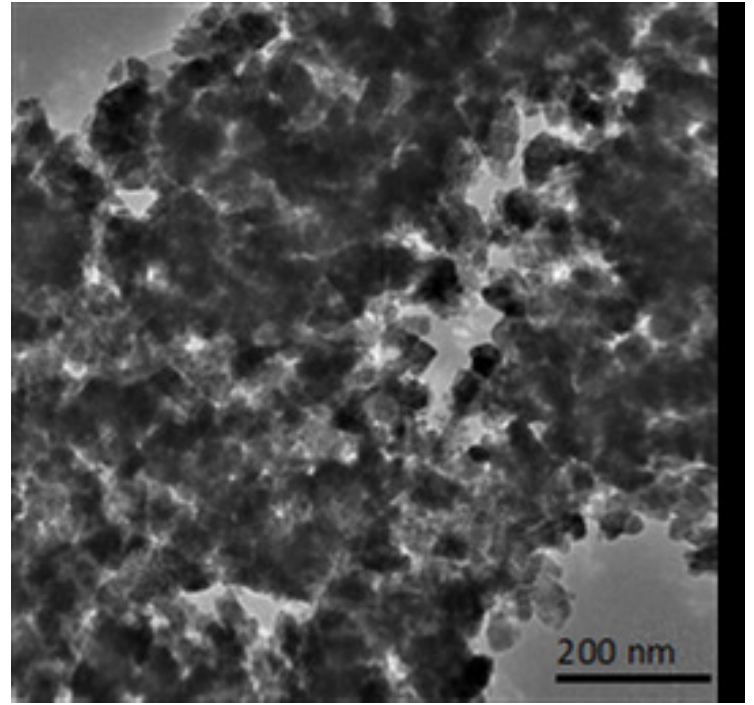

Figure 1. TEM images of Fe-ZnO nanoparticles.

sented as mean with standard deviation. For data analysis and graphs, OriginPro 2017 (Origin-Lab) software was used.

\section{Results and discussion}

\subsection{Characterization of $\mathrm{Fe}-\mathrm{ZnO}$ nanoparticles}

Morphology (size and shape) of Fe- $\mathrm{ZnO}$ nanoparticles was investigated using TEM (Fig. 1). The shape of synthesized Fe- ZnO nanoparticles was both square and hexagonal with an average size of $30 \mathrm{~nm}$. $\mathrm{Fe}-\mathrm{ZnO}$ also showed agglomeration, which is a common phenomenon in metal nanoparticles. Due to this feature, the nanoparticles were dispensed with the help of sonication when used in the photo-degradation experiment.

The crystalline structure of $\mathrm{Fe}-\mathrm{ZnO}$ nanoparticles from XRD (Fig. 2) showed diffraction peaks at $2 \theta$ i.e., $31.61^{\circ}, 34.26^{\circ}, 36.10^{\circ}, 47.37^{\circ}$, $56.40^{\circ}, 62.68^{\circ}$, and $67.72^{\circ}$ corresponding to (100), (002), (101), (102), (110), (103), and (112) planes, respectively, which specifies hexagonal wurtzite structure of Fe- $\mathrm{ZnO}$ nanoparticles. The peak broadening denoted that the synthesized structures were in the nano-range [40].

\subsection{Degradation of LDPE}

Treatment of LDPE films with Fe- $\mathrm{ZnO}$ clearly showed the occurrence of photocatalytic degradation, which was confirmed by the significant reduction in weight. A maximum weight loss of $13.8 \%$ for

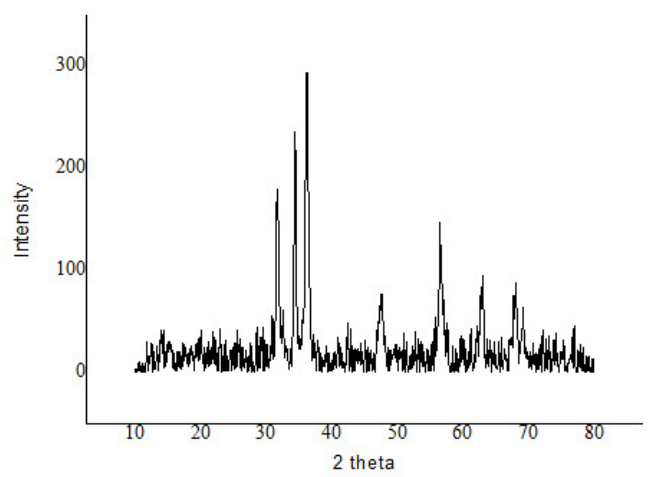

Figure 2. XRD of Fe-ZnO nanoparticles.

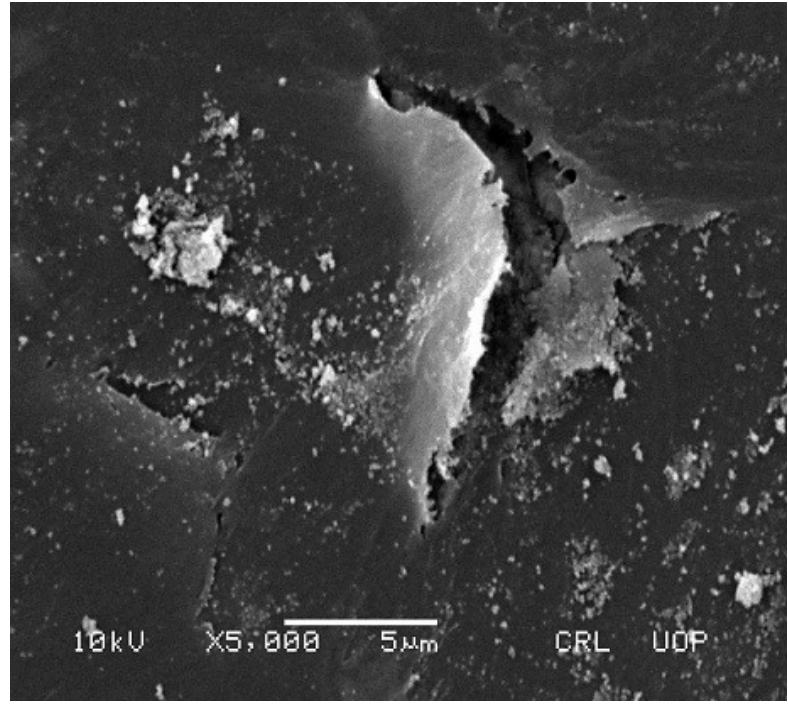

Figure 3. SEM image of degraded LDPE after 14 days.

pure LDPE films was observed at Fe-ZnO dose of $200 \mu \mathrm{g} / \mathrm{ml}$ while that of 100 and $10 \mu \mathrm{g} / \mathrm{ml}$ showed 9 and $5.7 \%$ degradation, respectively. In comparison, no weight reduction was observed in the dark and in the control.

Visual deformation of the LDPE films was also noticed including the formation of pores, shrinkage, and an increase in density. The deformation was confirmed by SEM analysis, which further revealed the appearance of fractures, dents, and surface roughness in the NP treated LDPE. Figure 3 shows the SEM images of degraded LDPE under light after 14 days. Interestingly, the white precipitates on the plastic surface showed the presence of nanoparticles. However, a major fraction of nanoparticles was also embedded in the LDPE matrix, which further accelerated the degradation process. It was observed that the nanoparticles were tightly bound to the LDPE matrix and couldn't be released upon sonication. This is an interesting aspect and requires further work to understand the underlying mechanism of such binding of inert nanoparticles to the plastic films.

From FTIR analysis (Fig. 4), different peaks were observed after NP treatment in the presence of light, which corresponded to the degradation of LDPE. The predominant groups at positions 1377, 1368, and $890 \mathrm{~cm}^{-1}$ for $\mathrm{CH}_{3}, 2915$ and $2845 \mathrm{~cm}^{-1}$ for $\mathrm{C}-\mathrm{H}$, and 1467 and 1462 $\mathrm{cm}^{-1}$ for $\mathrm{CH}_{2}$ [41] disappeared after treatment with the nanoparticles. The disappearance of these groups suggested the attack by ROS converting such groups to $\mathrm{O}-\mathrm{H}, \mathrm{C}=\mathrm{C}$, and $\mathrm{C} \equiv \mathrm{C}$ stretches, respectively, at peak positions 3278,1635 , and $2123 \mathrm{~cm}^{-1}$. These functional groups represented that they were converted to alcohols, alkenes, and alkynes as the degradation products of LDPE. However, in dark condition, no degradation products were found, which is clear evidence of ROS based degradation.
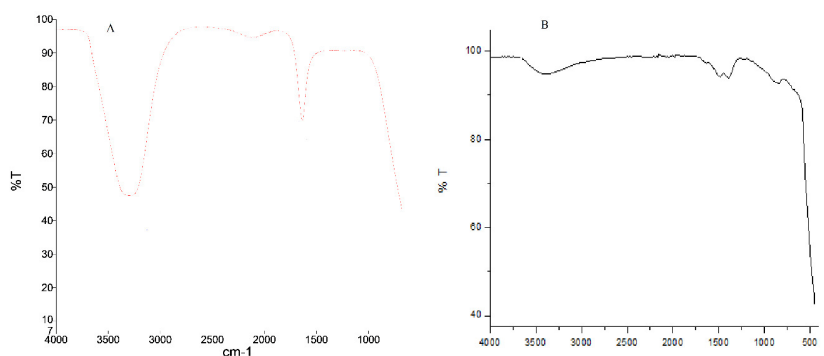

Figure 4. FTIR of sample solution after (A) and before (B) exposure 


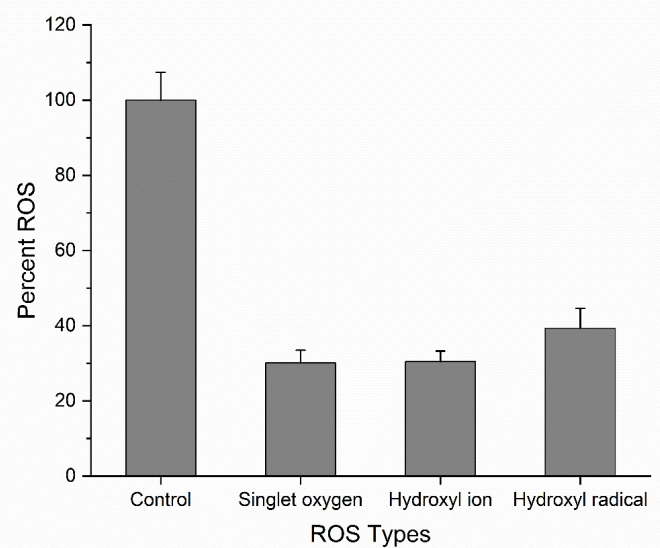

Figure 5. Percentage of the different ROS moieties generated by Fe-ZnO

\subsection{Fe-ZnO photo-activation}

The photocatalytic effect of $\mathrm{Fe}-\mathrm{ZnO}$ was evaluated through the quantification and identification of ROS produced after LED light exposure. For ROS quantification, DPBF fluorescent dye was used for singlet oxygen and hydroxyl radical quantification [42, 43]. ROS were produced due to photo-excitation of Fe- $\mathrm{ZnO}$ nanoparticles in ethanol using DPBF. The dye reacts with singlet oxygen and causes a decrease in the absorbance which was checked at $410 \mathrm{~nm}$ [16]. For quantum yield calculation, methylene blue (MB) was used as a standard photosensitizer. The natural logarithmic values from DPBF absorbance were plotted against irradiation time [16]. The quantum yield of $\mathrm{Fe}-$ $\mathrm{ZnO}$ nanoparticles was calculated using Eq. (2).

$$
\Phi_{\mathrm{FeZnO}}=\Phi_{\mathrm{MB}} \frac{\mathrm{K}_{\mathrm{FeZnO}}}{\mathrm{K}_{\mathrm{MB}}} \frac{I_{M B}}{I_{F e Z n O}}
$$

Where $\Phi_{\mathrm{FeZnO}}$ is the quantum yield of Fe- $\mathrm{ZnO}$ nanoparticles, $\Phi_{\mathrm{MB}}$ is the same of $\mathrm{MB}$ photosensitizer (i.e. 0.52) [16], $\mathrm{K}_{\mathrm{FeZnO}}$ and $\mathrm{K}_{\mathrm{MB}}$ are rate constants for the degradation of DPBF and standard MB photosensitizer, respectively, and $I_{F e Z n O}$ and $I_{M B}$ represent the photons absorbed by nanoparticles and MB standard, respectively. The quantum yield as a function of ROS generated from the surface of $\mathrm{Fe}-\mathrm{ZnO}$ was determined as $0.29 \mathrm{in}$ ethanol. The nanoparticles were synthesized in a way to incorporate more iron on the surface of $\mathrm{ZnO}$ (Fig. 5) for increased generation of ROS.

Such nanoparticles follow the type II photodynamic reaction which generates singlet oxygen. The process begins when the surface of $\mathrm{Fe}$ $\mathrm{ZnO}$ absorbs photons from the visible portion of the electromagnetic spectrum. This photoexcites the $\mathrm{Fe}-\mathrm{ZnO}$ into a singlet energy state, which is further converted to a triplet state. Afterward, this energy is transferred from the excited triplet state on $\mathrm{Fe}-\mathrm{ZnO}$ to the triplet ground state of oxygen which generates singlet oxygen. This singlet oxygen was calculated using scavengers such as sodium azide. However, The lifetime of singlet oxygen is very short and can further give rise to hydroxyl radicals and hydroxyl ions [44]. In the current study, the produced hydroxyl radicals and ions were calculated using scavengers like mannitol for hydroxyl radicals and DMSO for hydroxyl ions. However, there was no such ROS moieties generation in dark.

Figure 5 revealed that different ROS moieties were formed in various percentages pointing to their role in PE degradation. Interestingly, these ROS moieties are highly reactive and directly attack the surrounding organic matter, thus degrading PE. The ROS moieties were singlet oxygen $(30.11 \%), \mathrm{OH}$ ions $(30.45 \%)$, and $\mathrm{OH}$ radicals $(39.34 \%)$. The control showed the scavenging of all the ROS radicals $(100 \%)$ in the presence of sodium azide, mannitol, and DMSO.

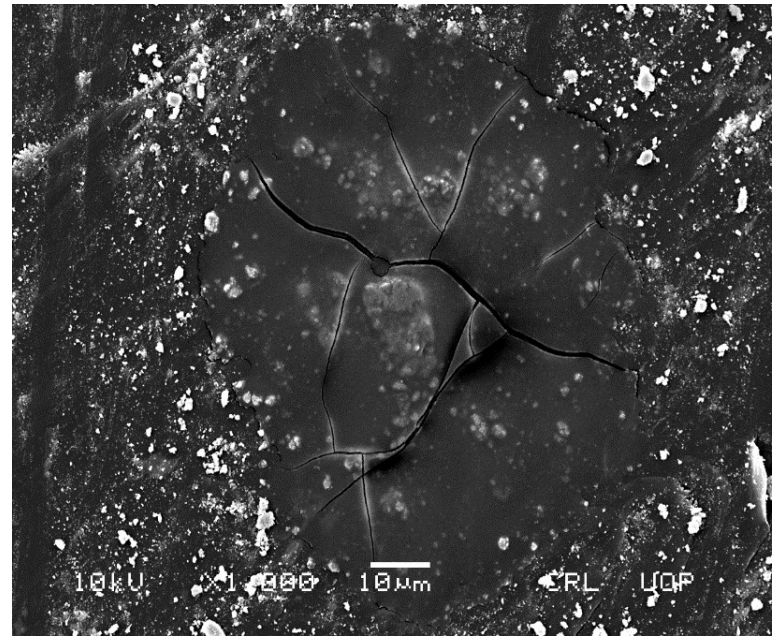

Figure 6. Surface morphology of the degraded commercial grade PE film

Previously, we reported that doped $\mathrm{ZnO}$ nanoparticles can generate heat while absorbing photons [37]. To confirm the occurrence of any photothermal phenomenon with $\mathrm{Fe}-\mathrm{ZnO}$, photothermal activity was assessed. Interestingly, no rise in temperature was observed for up to $30 \mathrm{~min}$, which confirmed the only role of ROS in the degradation of LDPE, further corroborated by the absence of any degradation in dark conditions.

\section{Photocatalytic degradation mechanism}

The produced ROS started the degradation of PE by attacking the polymer chain $[45,46]$. ROS also diffused through the PE matrix and degraded the inner matrix of the polymer. This was because of $\mathrm{C}$ $\mathrm{C}$ and $\mathrm{C}-\mathrm{H}$ bonds presence in PE. The ROS started the reaction by attaching to the $\mathrm{C}-\mathrm{C}$ and $\mathrm{C}-\mathrm{H}$ bonds and caused cleavage of these bonds [47] and formed carbonyl groups as intermediate. These carbonyl groups are in turn oxidized by $\mathrm{H}_{2} \mathrm{O}$ and $\mathrm{CO}_{2}$ with the help of ROS produced [45].

\section{Degradation of commercial-grade PE}

Degradation of commercial-grade PE films was investigated using 100,500 , and $1000 \mu \mathrm{g} / \mathrm{ml} \mathrm{Fe}-\mathrm{ZnO}$ doses (Fig. 6). Compared to pure LDPE, a higher nanoparticle dose was used in these experiments since degradation at lower nanoparticle doses was negligible. One of the possible reasons could be the presence of additives such as stabilizers and antioxidants in commercial PE bags [48]. With commercial PE, a maximum of $15 \%$ weight reduction was observed after 14 days at 1000 $\mu \mathrm{g} / \mathrm{ml}$ followed by $7.8 \%$ with $500 \mu \mathrm{g} / \mathrm{ml}$ and $1 \%$ with $100 \mu \mathrm{g} / \mathrm{ml} \mathrm{NP}$ doses. Comparatively, no weight reduction/degradation was observed in the dark and the control without $\mathrm{Fe}-\mathrm{ZnO}$ addition. These results were in agreement with those observed with pure LDPE and therefore follow the same degradation route with an active role of ROS.

\section{Conclusions}

Degradation of $\mathrm{PE}$ by $\mathrm{Fe}-\mathrm{ZnO}$ was concentration as well as lightdependent (generating ROS from the surface of the nanoparticle) since no degradation was observed in the dark. It is inferred that higher degradation may be achieved by increasing $\mathrm{Fe}-\mathrm{ZnO}$ concentration and reaction time. Volatile organic products generated during photo-degradation produced defects in the films which were confirmed by FTIR, SEM, and weight reduction analysis. FTIR confirmed the formation of the $\mathrm{C}=\mathrm{C}, \mathrm{C} \equiv \mathrm{C}$, and $\mathrm{OH}$ groups, which showed that different compounds were formed after degradation such as ethylene, acetylene, ethanol, phenol, carboxylic acid, etc. Further, doping changed 
the activation range of $\mathrm{Fe}-\mathrm{ZnO}$ from $\mathrm{UV}$ to visible light whereas the excitation is also dependent on the quantity and type of dopant used. Therefore, the ratio of dopant can be changed to enhance degradation. With activation in visible light, Fe- $\mathrm{ZnO}$ offers an attractive substitute method to degrade plastic in the outdoor environment.

\section{Declaration}

The authors declare no competing financial interest.

\section{References}

[1] J. Arutchelvi, M. Sudhakar, A. Arkatkar, M. Doble, S. Bhaduri, and P. V. Uppara, Indian J. Biotech. 7, 9 (2008).

[2] A. Chamas, H. Moon, J. Zheng, Y. Qiu, T. Tabassum, J. H. Jang, M. A. Omar, S. L. Scott, and S. Suh, ACS Sustainable Chem. Eng. 8, $3494(2020)$.

[3] J. A. Glaser, Plastics in the Environment Chapter 5, 73 (IntechOpen, 2019).

[4] G. Gourmelon, Worldwatch Institute (2015).

[5] M. Hikmah, R. Setyaningsih, and A. Pangastuti, IOP Conf. Ser.: Mater. Sci. Eng. 333, 012076 (IOP Publishing Ltd, 2018).

[6] A. Kapri, M. Zaidi, and R. Goel, J. Microbiol. Biotechnol. 20, $1032(2010)$.

[7] M. Bhatia, A. Girdhar, B. Chandrakar, and A. Tiwari, J. Nanomed. Nanotechnol. 4, 1000175 (2013).

[8] R. Sarker, P. Chakraborty, P. Paul, A. Chatterjee, and P. Tribedi, Arch Microbiol 202, 2117 (2020).

[9] B. M. Kyaw, R. Champakalakshmi, M. K. Sakharkar, C. S. Lim, and K. R. Sakharkar, Indian J. Microbiol. 52, 411 (2012).

[10] A. Sangroniz, J. B. Zhu, X. Tang, A. Etxeberria, E. Y. X. Chen, and H. Sardon, Nat. Commun. 10, 3559 (2019).

[11] A. Gajendiran, S. Krishnamoorthy, and J. Abraham, 3 Biotech 6, $52(2016)$

[12] J. Abraham, E. Ghosh, P. Mukherjee, and A. Gajendiran, Environ. Prog. Sustain. 36, 147 (2017).

[13] S. H. Kim, S. Y. Kwak, and T. Suzuki, Polymer 47, 3005 (2006).

[14] P. Kamalian, S. N. Khorasani, A. Abdolmaleki, M. Karevan, S. Khalili, M. Shirani, and R. E. Neisiany, J. Polym. Eng. 40, 181 (2020).

[15] J. Montanari, C. Maidana, M. I. Esteva, C. Salomon, M. J. Morilla, and E. L. Romero, J. Control. Release 147, 368 (2010).

[16] L. Xiao, L. Gu, S. B. Howell, and M. J. Sailor, ACS Nano 5, 3651 (2011).

[17] B. Khurana, P. Gierlich, A. Meindl, L. C. Gomes-da-Silva, and M. O. J. P. Senge, Photochem. Photobiol. Sci. 18, 2613 (2019).

[18] A. Nadhman, S. Nazir, M. I. Khan, S. Arooj, M. Bakhtiar, G. Shahnaz, and M. Yasinzai, Free Radical Bio. Med. 77, 230 (2014).

[19] A. Kulkarni and H. Dasari, MATEC Web Conf. 144, 02023 (2018).

[20] A. Nadhman, M. I. Khan, S. Nazir, M. Khan, G. Shahnaz, A. Raza, D. F. Shams, and M. Yasinzai, Int. J. Nanomedicine 11, 2451 (2016).

[21] R. T. Thomas and N. Sandhyarani, RSC Adv. 3, 14080 (2013).

[22] W. Asghar, I. A. Qazi, H. Ilyas, A. A. Khan, M. A. Awan, and M. Rizwan Aslam, J. Nanomater. 2011, 461930 (2011).

[23] P. A. Zapata, F. M. Rabagliati, I. Lieberwirth, F. Catalina, and T. Corrales, Polym. Degrad. Stab. 109, 106 (2014).

[24] P. Kamalian, S. N. Khorasani, A. Abdolmaleki, and R. E. Neisiany, Polym. Bull. 76, 3593 (2019).

[25] V. M. Pathak and N. Kumar, Energ. Ecol. Environ. 2, 418 (2017)

[26] S. S. Ali, I. A. Qazi, M. Arshad, Z. Khan, T. C. Voice, and C. T. Mehmood, Environ. Nanotechnol. Monit. Manage. 5, 44 (2016).

[27] S. Chakrabarti, X. Liu, C. Li, P. Banerjee, S. Maitra, and M. T. Swihart, Int. J. Mater. Eng. Innov. 6, 18 (2015).
[28] G. T. Mazitova, K. I. Kienskaya, D. A. Ivanova, I. A. Belova, I. A. Butorova, and M. V. Sardushkin, Rev. J. Chem. 9, 127 (2019).

[29] X. Wang, Y. Ding, C. J. Summers, and Z. L. Wang, J. Phys. Chem. B. 108, 8773 (2004).

[30] M. Farahmandjou and S. Jurablu, Int. J. Bio-Inorg. Hybrid Nanomater. 3, 179 (2014).

[31] S. George et al., ACS Nano 4, 15 (2009).

[32] M. Carofiglio, S. Barui, V. Cauda, and M. Laurenti, Appl. Sci. 10, 5194 (2020).

[33] T. Srinivasulu, K. Saritha, and K. T. R. Reddy, Mod. Electron. Mater. 3, 76 (2017).

[34] P. D. Phonsy, S. G. Anju, K. P. Jyothi, S. Yesodharan, and E. P. Yesodharan, J. Adv. Oxid. Technol. 18, 85 (2015).

[35] R. Hong, T. Pan, J. Qian, and H. Li, Chem. Eng. J. 119, 71 (2006).

[36] S. S. Kumar, P. Venkateswarlu, V. R. Rao, and G. N. Rao, Int. Nano Lett. 3, 30 (2013).

[37] A. Nadhman, S. Nazir, M. I. Khan, A. Ayub, B. Muhammad, M. Khan, D. F. Shams, and M. Yasinzai, Int. J. Nanomed 10, 6891 (2015).

[38] A. Islam et al., Nanomedicine (Lond) 15, 755 (2020).

[39] J. D. Blando, R. J. Porcja, and B. J. Turpin, Aerosol Sci. Tech. 35, 899 (2001).

[40] A. C. Mohan and B. Renjanadevi, Procedia Technology 24, 761 (2016).

[41] M. R. Jung et al., Mar. Pollut. Bull. 127, 704 (2018).

[42] Y. Okada and H. Okajima, Yakugaku Zasshi 118, 226 (1998).

[43] R. Vankayala, C. C. Lin, P. Kalluru, C. S. Chiang, and K. C. Hwang, Biomaterials 35, 5527 (2014).

[44] W. M. Sharman, C. M. Allen, and J. E. V. Lier, Drug Discov. Today 4, 507 (1999).

[45] X. Zhao, Z. Li, Y. Chen, L. Shi, and Y. Zhu, Appl. Surf. Sci. 254, 1825 (2008)

[46] M. Mucha, S. Bialas, and H. Kaczmarek, J. Appl. Polym. Sci. 131, 40144 (2014).

[47] P. Mormile, L. Petti, M. Rippa, B. Immirzi, M. Malinconico, and G. Santagata, Polym. Degrad. Stab. 92, 777 (2007).

[48] Y. Gao, Y. Gu, and Y. Wei, J. Agric. Food Chem. 59, 12982 (2011). 